\title{
ISSUES IN IMPLEMENTING THE KIRCHHOFF INTEGRAL FOR 3D PRE-STACK DEPTH MIGRATION
}

BOB GODFREY and KJELL BERG Schlumberger/Geco-Prakla, Schlumberger House, Buckingham Gate, Gatwick Airport, West Sussex RHG ONZ, UK

The Kirchhoff approach to depth migration requires computation of travel times. We use dynamic ray tracing to shoot a bundle of rays upward from each subsurface point to be imaged. The number of rays varies between a few to several thousand rays per bundle and is dependent on the local complexity of the velocity-depth model. For instance, in sedimentary sections, 1500 rays may be sufficient to accurately determine travel times. However, in the presence of salt structures, up to 7000 rays may be required. For large-scale problems associated with complex structures, implementation of 3-D prestack depth migration requires efficient construction of travel time tables and their use in Kirchhoff summation.

Two issues that affect the design of the Kirchhoff operator are (i) operator aliasing and (ii) operator weighting. Most 3-D surveys have cross-line bin spacing ranging from 25 to 50 meters. This causes severe aliasing of high frequencies unless properly accounted for in the operator design. We use continuously time-varying low-pass filters in an attempt to minimize the artifacts caused by aliasing. In our implementation, operator weights are used to account for divergence and shotgeophone obliquity factors. This means that amplitude variations along the operator caused by caustics and multi-pathing are not accounted for as they would be using a wave theoretic approach.

We have implemented the 3-D prestack depth migration using a massively parallel processor. In practise, this has enabled us to achieve fast turnaround without compromising the fidelity aspects of the algorithm discussed above. Figure (1) shows the result of prestack depth migrating 3.5 million input traces to image a 1024 trace target line, $6 \mathrm{~km}$ in depth.

3-D prestack depth migration can be a successful tool for verifying accuracy of layer velocities used to define macro velocity-depth models. Figure (2) shows a set of image gathers selected from the previous figure. Curvature of events seen on image gathers can then be used to verify layer velocities derived from, for example, prestack travel time inversion.

In the presentation, we shall report the results of our study on the issues in implementing the Kirchhoff integral for 3-D prestack depth migration and demonstrate the use of image gathers using Gulf of Mexico and North Sea data examples. 
3-0 Prestact Kirchofit Depth Migration

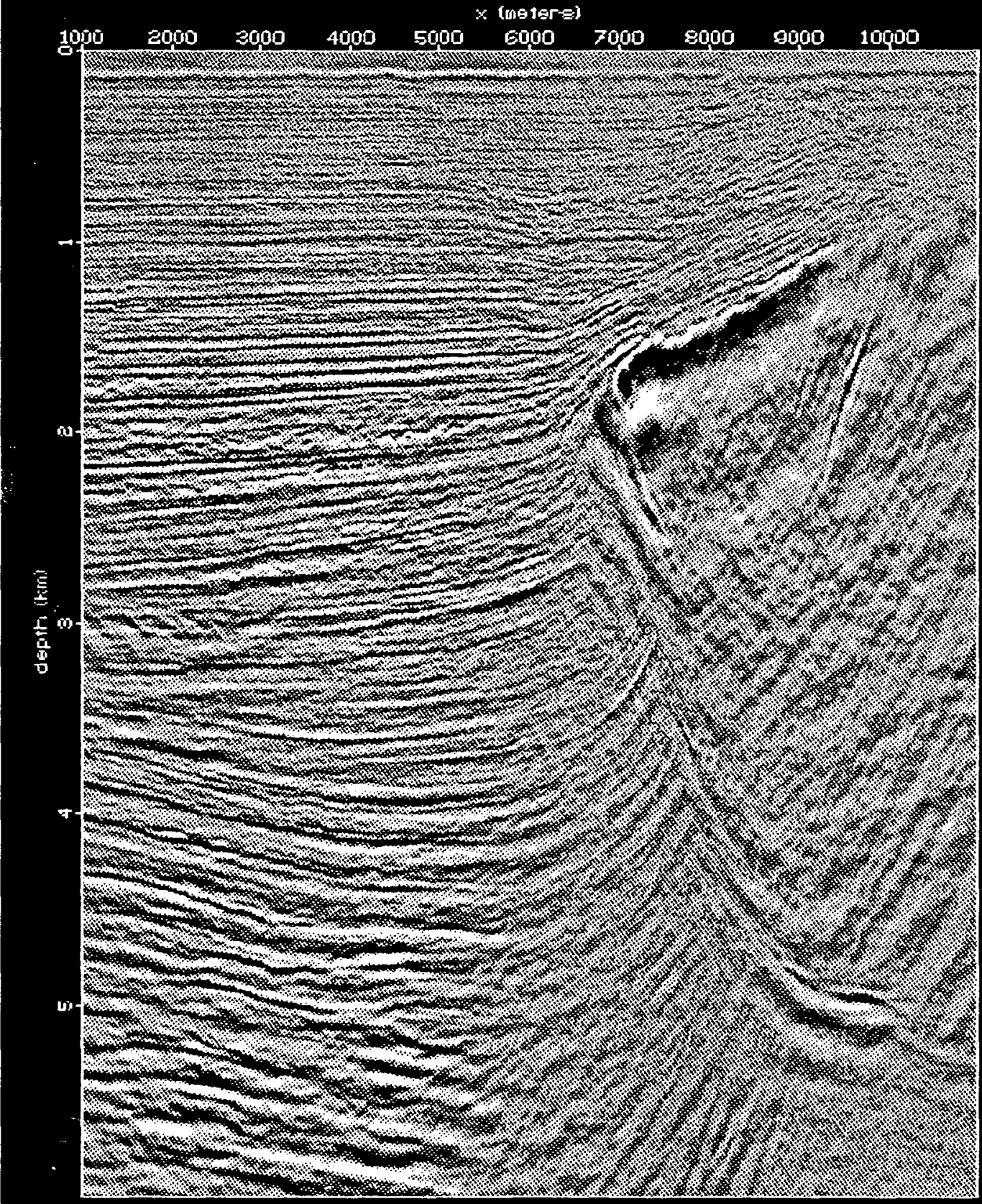

Figure 1: 3-D prestack depth migration outputting a line targeted around a salt dome in Vermilion. Area 2, offshore Louisiana. 


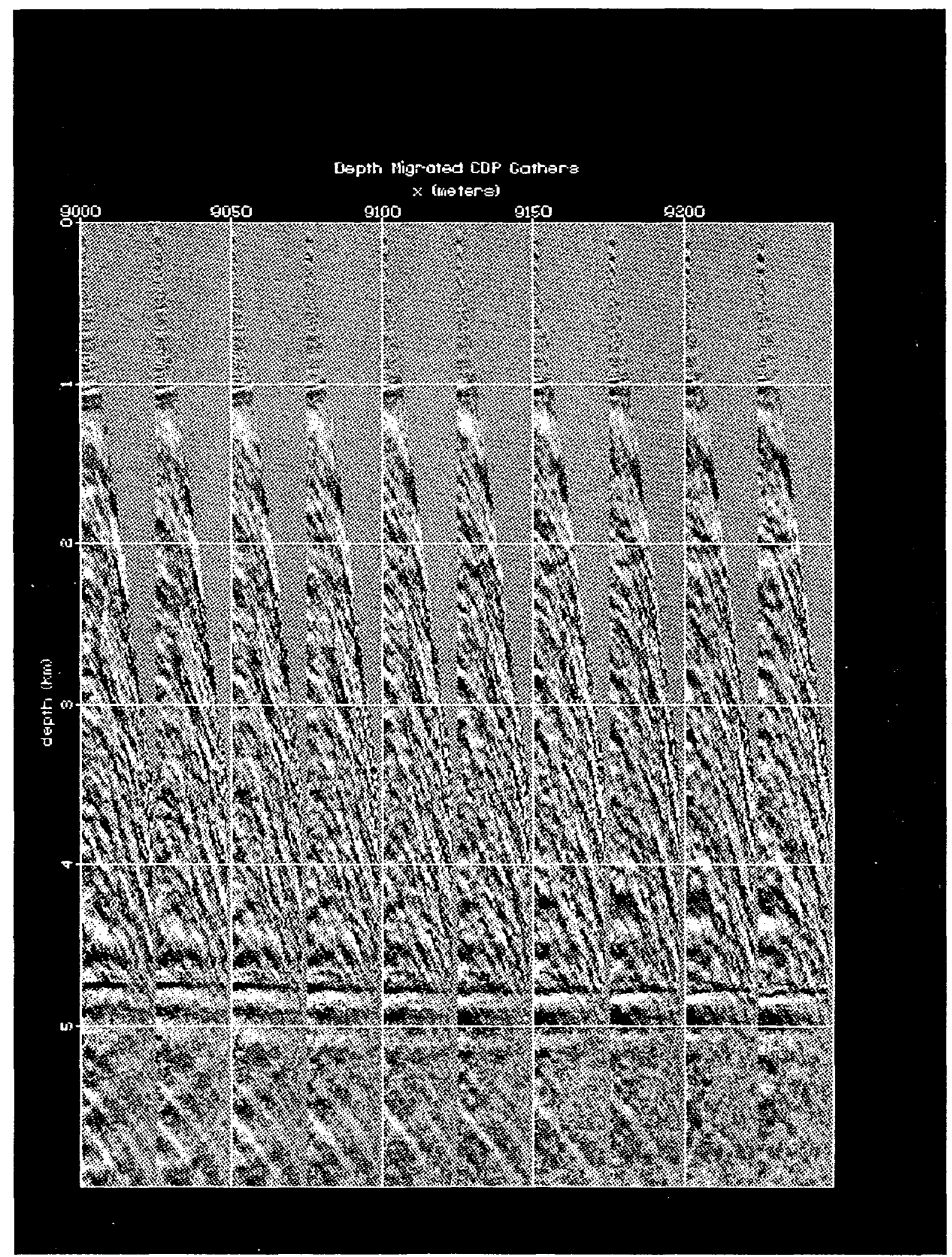

Figure 2: Common depth point gathers selected at $\mathrm{x}=9 \mathrm{~km}$ from previous figure. The top salt reflector exhibits upward curvature indicating the background velocity field is too low at this location. Note the strong base of salt reflector. 
